\title{
Compte rendu d'ouvrage
}

\section{Sous la direction de Martine Berger et Jean-Louis Chaléard, Villes et campagnes en relations, regards croisés Nords-Suds, Karthala, 2017, 299 p.}

\author{
Guillaume LACQUEMENT \\ Département de Géographie et d'Aménagement \\ UMR 5045 ART-Dev, Acteurs, Ressources, Territoires dans le Développement \\ Université de Perpignan Via Domitia \\ lacqueme@univ-perp.fr
}

L'ouvrage compile une sélection de textes issus d'un colloque du labex Dynamite qui en juin 2015 a présenté les principaux résultats d'une étude consacrée à la reconfiguration des rapports villescampagnes dans le contexte de la métropolisation et de la globalisation. Prenant le parti d'une réflexion critique sur les catégories et les modèles, il propose une analyse comparative des relations fonctionnelles et politiques entre villes et campagnes. Il met en miroir les pays des Nords et ceux des Suds pour caractériser les relations de complémentarités et d'échanges produites par les mobilités résidentielles et de travail, la diversification des activités socio-économiques, les préoccupations environnementales et les nouvelles demandes sociales. Il fait le pari de la pertinence de la distinction Nord-Sud pour considérer les contextes, les processus et les temporalités qui différencient la dynamique contemporaine des systèmes villes-campagnes.

Après avoir retracé dans deux chapitres distincts, l'un consacré aux pays des Nords, l'autre à ceux des Suds, les principales étapes de la production du savoir géographique sur les relations villescampagnes, pour mettre en perspective l'évolution des catégories d'analyse de la discipline, les auteurs organisent l'ouvrage en quatre parties. La première (Systèmes d'activités et mobilités entre villes et campagnes) s'intéresse au rôle que jouent les échanges et les complémentarités dans l'accès aux ressources et dans les stratégies de reproduction sociale des individus. Elle interroge les itinéraires migratoires, les types de polarisation des flux, les formes nouvelles de multirésidentialité, les politiques publiques d'aménagement pour comprendre l'évolution structurelle et spatiale des activités socio-économiques et la dynamique des systèmes urbains. La lecture post-coloniale des mobilités kanakes en Nouvelle-Calédonie retient l'intérêt pour la réflexion que les auteurs proposent sur la pertinence des modèles interprétatifs de l'organisation de l'espace insulaire et de ses pratiques habitantes. La deuxième partie considère les espaces périurbains dans la diversité de leurs configurations spatiales pour caractériser les formes de contact et d'hybridation à travers le prisme des maillages territoriaux, des systèmes de gouvernance et des orientations de la politique d'aménagement du territoire. Les contributions sont denses en informations et on apprécie pour certaines la manière dont la démarche empirique a été mobilisée et dont ses résultats sont restitués, en particulier pour comprendre comment les projet d'aménagement fabriquent du territoire dans l'espace périurbanisé. Dans la troisième partie (agriculture et proximité, nourrir les métropoles), les enjeux de l'approvisionnement alimentaire des métropoles sont saisis pour étudier les formes ambivalentes de transformation de l'activité agricole. Le chapitre souligne les effets complexes de cette transformation sous influence urbaine sur les structures agraires et la multifonctionnalité de l'agriculture. Certaines des contributions évoquent en outre les inégalités nouvelles qu'elle fait naître chez les producteurs agricoles et celles qu'elle traduit au sein de la demande urbaine. Enfin, la dernière partie (politiques publiques et mutations des relations villes-campagnes) montre de quelle manière les catégories spatiales du rural, de l'urbain et du périurbain sont interprétées par les acteurs politiques dans la 
prospective territoriale et l'action d'aménagement, et à quel point cette rhétorique traduit la vision politique de l'organisation sociale de l'espace. La conclusion est bienvenue pour rendre plus intelligible l'articulation des quatre chapitres dépourvus de paragraphes introductifs et pour évoquer les apports des regards croisés nords-suds à la connaissance et à la discipline géographiques.

Martine BERGER, Jean-Louis CHALEARD, éds, Villes et campagnes en relations, regards croisés Nords-Suds, Karthala, 2017, 299 p., in Annales de Géographie, n727, 2019, p.116. 